Title: Antenatal vaccine uptake - a cross-sectional study investigating factors influencing women's choices in pregnancy

\title{
Authors:
}

Dr Drew MOIR (corresponding author)

House Medical Officer in Obstetrics and Gynaecology, Western Health

Postal Address: 33 Epsom Road, Kensington 3031

Email: Drew.Moir@wh.org.au

Phone: 0437197456

Dr Konrad GUNTER

House Medical Officer, Western Health

Email: Konrad.Gunter@wh.org.au

Ms Lee-Anne LYNCH

Clinical Research Midwife, Maternal Fetal Medicine department, Western Health

Email: Lee-Anne.Lynch@wh.org.au

Ms Sara VOGRIN

Biostatistician, Western Health, Melbourne Medical School, The University of Melbourne

Email: sara.vogrin@unimelb.edu.au

Associate Professor Joanne SAID

Head of Maternal Fetal Medicine, Western Health

Email: jsaid@unimelb.edu.au

\section{Study Location:}

Western Health (Sunshine Hospital) Maternal Fetal Medicine Department

175 Furlong Road

St Albans, 3021

\section{Acknowledgements}

The authors have no conflicts of interest to declare.

The authors would like to thank partnerships with the University of Melbourne and Western Health for their support in the completion of this research. The authors would also like to thank Jenny Patterson for her role in translation services liaison and Irene Bryan for her assistance with proof-reading and editing

This is the author manuscript accepted for publication and has undergone full peer review but has not been through the copyediting, typesetting, pagination and proofreading process, which may lead to differences between this version and the Version of Record. Please cite this article as doi: $10.1111 / \mathrm{AJ} 0.13146$

This article is protected by copyright. All rights reserved 
Article type : Original Manuscript

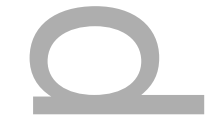

0

Antenatal vaccine uptake - a cross-sectional study investigating factors influencing women's choices in pregnancy

Antenatal Vaccine Uptake - A cross-sectional study

Abstract word count: 244

Main text word count: 2488

List of Figures

1. Figure 1. Rates of vaccination breakdown by month of birth

2. Figure 2. Thematic breakdown of vaccination decisions

List of Tables

1. Table 1. Socio-demographic characteristics

2. Table 2. Summary statistics and logistic regression of participants by vaccine status

Keywords: maternal, antenatal, vaccination, influenza, pertussis

\section{Abstract}

Background: Seasonal influenza and pertussis cause significant morbidity and mortality among expectant mothers and infants. Vaccination during the antenatal period is an important public health intervention, minimising rates of maternal, neonatal and infant infection.

Aims: The primary aim of this project was to establish the rates of antenatal vaccine uptake. Secondly, the study aimed to determine socio-demographic factors significant to vaccine uptake. Thirdly, the project aimed to produce a thematic analysis of the factors affecting vaccination uptake during pregnancy. Materials and Methods: A cross-sectional observational study was conducted amongst women attending a large maternity hospital, in the western suburbs of Melbourne, for perinatal care. Data was collected via selfcompleted questionnaires after delivery. Data from the questionnaires was entered into an electronic database and STATA was used to undertake correlation analysis. 
Results: Over a twelve-month period 1678 women completed questionnaires and 1305 were eligible for further analysis. The uptake of influenza vaccine uptake was $48.3 \%$, pertussis vaccine uptake was higher, at 82.9\%. Uptake of influenza and pertussis vaccines strongly correlated with recommendations from healthcare providers (OR 29.7 and 63.8 respectively). Maternal country of birth, age and parity were significant predictors of vaccine uptake. In thematic analysis, healthcare provider recommendation and the perceived risk of the disease were factors resulting in vaccination.

Conclusion: This study determined the rate of antenatal vaccine uptake and significant socio-demographic determinants affecting uptake at a large maternity hospital in metropolitan Melbourne. Ensuring healthcare providers recommend vaccination is likely to improve coverage.

\section{Introduction}

Bordetella pertussis causes a significant burden of disease in infant populations globally ${ }^{(1-3)}$. Australia is one of a number of developed countries experiencing a resurgence of pertussis disease following the change from whole-cell vaccine $(\mathrm{wP})$ to acellular vaccine $(\mathrm{aP})$ and ongoing microbial adaptation ${ }^{(4,5)}$. The most

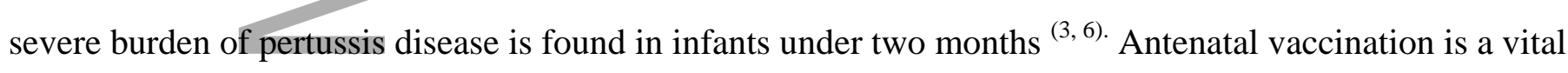
strategy to protect these most at risk infants. Immunisation against pertussis with the combined diphtheriatetanus-acellular pertussis vaccine has $90 \%$ efficacy in preventing new cases of the disease in neonates ${ }^{(7)}$. Pregnant women and neonates are a priority group for influenza vaccination as per World Health Organisation (WHO) guidelines as they are at increased risk of morbidity and mortality from influenza illness. Infection can lead to pregnancy complications including stillbirth, pre term birth and fetal growth restriction $^{(8)}$. In Australia the hospitalisation rate for influenza in children under 5 months is 192 per $100,000 .{ }^{(9)}$ Vaccination against influenza has been shown to have $36 \%$ efficacy in preventing the clinical syndrome of respiratory illness with fever in pregnant women in addition to a reduction in clinic visits, and prevents approximately one third of febrile illness in infants up to 5-6 months of age ${ }^{(10)}$.

Vaccination against influenza and pertussis in the antenatal period is a safe and effective method of disease prevention. WHO position papers on seasonal influenza and aP pertussis vaccinations found that the vaccines are safe in pregnancy with no significant adverse reactions or perinatal complications ${ }^{(4,8)}$. Influenza vaccination in pregnancy was added to the National Immunisation Program in 2010, pertussis vaccination in third trimester of pregnancy was added in $2015^{(1)}$.

Despite the evidence supporting vaccine safety and efficacy, and development of national immunisation programs the uptake has been suboptimal in the antenatal setting ${ }^{(11,12)}$. A recent large Victorian study found 
antenatal uptake of seasonal influenza and pertussis vaccination to be $39 \%$ and $64 \%$ respectively. ${ }^{(13)}$ Factors contributing to low uptake include lack of recommendation by a health care professional, ethnic minorities and/or migrant status, perception of low disease risk, poor access and a belief that the vaccine is unsafe or ineffective ${ }^{(11,13,14)}$. Understanding and addressing these factors with easily accessible and culturally appropriate immunisation programs may improve preventative healthcare.

Current evidence regarding antenatal vaccine uptake among ethnic minorities and migrant women is conflicting $^{(13-18)}$, further research in this area is needed.

The primary aim of this cross-sectional observational study was to determine the rate of antenatal vaccine uptake for women attending a large maternity hospital in metropolitan Melbourne for perinatal care. The project also sought to identify how vaccine uptake may be affected by socio-demographic factors in a multiculturally and linguistically diverse patient population. Lastly, the study aimed to better understand women's decision-making regarding vaccination during pregnancy.

\section{Materials and Methods}

A convenience sample was recruited from the maternity wards of a large hospital in Melbourne's western suburbs. We aimed for a large sample size to allow detection of differences between minority groups. Women who delivered prior to 32 weeks' gestation, were unwell, experienced a traumatic delivery or stillbirth were considered ineligible and excluded.

Ethical approval was granted by the Western Health Low Risk Human Research Ethics Panel (LREP Project Number: LNR/16/WH/185) in accordance with the requirements of the National Statement on Ethical Conduct in Human Research (2007).

Participants were approached on days 1-3 after delivery. Participants were provided with a Patient Information statement and consented into the study.

Participants completed a self-administered questionnaire in paper format, with recruitment occurring Monday-Friday. Recruitment numbers were higher in the initial four months of the study due to availability of dedicated research personnel. Participants were offered questionnaires in English, Vietnamese, Burmese, Punjabi, Arabic or Simplified Chinese (the most commonly utilised translation services within the hospital). Completed questionnaires were entered into an electronic database. Incomplete questionnaires, and questionnaires in which the answer to one question contradicted another, were excluded from further analysis.

A woman's region of origin was determined by using the United Nations geoscheme ${ }^{(19)}$. Factors that women identified as having an impact on their decision to vaccinate were recoded into thematic categories for analysis using Braun and Clarkes (2006) 6-step framework ${ }^{(20)}$. Open-ended responses were formatted into distinct variables and included in the thematic analysis.

STATA/IC14.2 was used for data analysis and statistical significance testing. Response frequency distributions were tabulated for each question. Fisher's Exact Test was utilised to investigate the differences in demographic variables between vaccinated and non-vaccinated women. Univariate and multivariate 
logistic regression were used to determine socio-demographic characteristics that significantly affected vaccine uptake.

\section{Results}

During the 12-month study period (23/01/2017 - 24/01/2018), 5572 women delivered live-born babies. Of these 5533 were delivered at a gestation over 32 weeks. A total of 3912 were eligible for participation in the study but 1165 were discharged prior to being approached by a researcher or were unable to access an appropriately translated questionnaire, thus 2747 women were invited to participate of whom 1678 consented and returned questionnaires. Unfortunately, 373 returned questionnaires had to be excluded from further analysis due to missing fields or contradicting responses, where the answer to one question contradicted another, thus 1305 questionnaires were included for analysis with a response rate $33.4 \%$. Table 1 presents the socio-demographic characteristics of study participants and the background population (all women with live births above 32 weeks gestation within the same time period).

Women completing the survey were found to have similar demographic characteristics to the background population of potentially eligible pregnant women, although women of African background were underrepresented (4.8 vs. $8.7 \%$ ) and women delivering at a gestation between 32-34 weeks were overrepresented (3.6\% vs. $1.0 \%)$.

Australian women were the largest group (42.9\%), comprising less than half of all those surveyed. Women from Southern and South-Eastern Asia constituted the next largest demographic (36.0\%). The vast majority of women opted to complete the questionnaire in English (97.6\%).

\section{Vaccine Uptake Rates}

The uptake of seasonal influenza vaccine (48.3\%) was less than pertussis (82.9\%). $72.9 \%$ of women reported receiving a recommendation for influenza vaccination, and 91.9\% a recommendation for pertussis vaccination.

Figure 1 depicts the breakdown of vaccination uptake by month of birth. Uptake of seasonal influenza vaccination varied greatly from $26.6 \%$ (those who gave birth in April) to $86.5 \%$ (those who gave birth in September). Uptake of pertussis was more consistent across the 12 month period, with the lowest uptake of pertussis vaccination update of 76.6\% (those who gave birth in April) and the highest was 97.6\% (those who gave birth in July).

\section{Factors significant to seasonal influenza vaccine uptake}

Table 2 describes the relationship between socio-demographic factors and influenza vaccine uptake.

Recommendation from a healthcare provider was the single factor most likely to predict a woman's influenza vaccination status. Women who received a recommendation had more than twenty-five times higher odds of receiving the influenza vaccine than those who did not (aOR 26.59; 95\%CI 16.23 - 43.52). 
Migration status was a predictor of influenza vaccine uptake, with migrant women having twice the odds of receiving the influenza vaccine as Australian women (aOR 2.13; 95\%CI 1.61 - 2.83). Receiving written information and receiving the pertussis vaccine both correlated positively with receiving the influenza vaccine.

\section{Factors significant to pertussis vaccine uptake}

Table 2 describes the relationship between socio-demographic factors and the uptake of pertussis vaccine.

Women who received a recommendation from a healthcare provider had more than fourty times higher odds of receiving the pertussis vaccine than those who received no recommendation (aOR 41.78; $95 \%$ CI $20.03-87.17)$.

Women aged under 25 years and over 45 years were less likely to receive the pertussis vaccine. Primiparous women were more likely to receive the pertussis vaccine than women having their second or subsequent child.

Unlike for influenza vaccination, migrant women were less likely to receive a pertussis vaccination compared with Australian women (aOR 0.50; 95\%CI $0.33-0.75$ ).

Receiving written information and receiving the influenza vaccine both correlated positively with receiving the pertussis vaccine.

\section{Self-identified barriers and facilitators of vaccination}

Figure 2 a depicts the thematic categories that facilitated vaccination in pregnancy. Health care provider (HCP) recommendation and women perceiving themselves or their infants to be at risk were the most prevalent reasons identified. Women cited recommendation from midwives and general practitioners more than obstetricians.

Positive messages from non-HCP sources such as family, friends and media were less commonly identified.

Figure $2 \mathrm{~b}$ demonstrates the thematic barriers to antenatal vaccination. Poor knowledge regarding vaccine efficacy, safety, current recommendations and potential perinatal morbidity of influenza/pertussis, and receiving insufficient recommendation and information from healthcare workers or other sources (insufficient positive information) were the most prevalent barriers to vaccination. Not normally receiving the influenza vaccine annually was the most common reason women cited for not receiving the vaccine (39.2\%). Seasonality of the influenza vaccine and system barriers were also common reasons for not receiving the vaccine antenatally.

Women commonly declined pertussis vaccination due to lack of time, also of note was the proportion of women who believed they did not need to receive the vaccine as they had received it in the past (16.1\%). 
The majority of women who did not receive the influenza and pertussis vaccine asserted that if they had received a recommendation from a HCP, they would have been influenced to accept the vaccines (68.3\% and $58.9 \%$ respectively)

\section{Discussion}

The results of this study demonstrate that the majority of pregnant women are not receiving the seasonal influenza vaccine. This is consistent with the findings of previous research; few study populations have managed to achieve coverage greater than $60 \%{ }^{(21)}$. Uptake of pertussis vaccine was higher with $82.9 \%$ of pregnant women receiving the vaccine. This is higher than other recent Victorian studies demonstrating uptakes of $64 \%$ and $70 \%(13,22)$.

During the period of this study a vaccination clinic co-located with the Antenatal Clinic was commenced 6 months into the 12 month study enabling access to vaccination at the point of antenatal care. This may have contributed to overall high rates of vaccination and an increase in vaccination uptake from January - June (82.2\% for pertussis, $46.0 \%$ for influenza) to with July - December (92.4\% for pertussis, $66.7 \%$ for influenza).

We hypothesise that the recommendation of healthcare providers may explain the difference in uptake rates between the two vaccines. Reports of recommendations for pertussis vaccines $(92.0 \%)$ were more prevalent than for seasonal influenza (73.0\%). This suggests that pregnant women are more aware of the benefits and importance of pertussis vaccination. A greater proportion of women demonstrated poor knowledge of the influenza vaccine, and received insufficient recommendation, compared with pertussis vaccine. Lastly, seasonal variation in vaccine availability and HCP awareness of the influenza season (AprilOctober) likely contributed to the lower uptake of influenza vaccine uptake and variability across the 12month period compared with pertussis vaccine uptake.

This study found that maternal age, parity and migrant status were variably associated with antenatal vaccine uptake. Multiparous women were less likely to receive the pertussis vaccine. This may be explained in part by the $16.3 \%$ of women who reported that they did not receive the vaccine as they had received it previously and were unaware they should receive it again.

Unexpectedly, this study found that women of migrant backgrounds were more than twice as likely as Australian women to receive the influenza vaccine but $50 \%$ less likely to receive the pertussis vaccine. Inconsistency has been a common theme in literature examining vaccine uptake among migrant and ethnically diverse women, some studies have shown no difference compared with Australian born women $(14,18)$, while other studies have found that women from ethnic minorities have lower rates of vaccine uptake ${ }^{(13,17)}$. Previous studies hypothesised that low uptake amongst migrant women may be due to cultural and language barriers. The high percentage of women opting to complete a questionnaire in English, even when translations were available, suggests that a language barrier is not a significant issue for this population. 
Future research may endeavour to further explore the different perceptions of vaccination between migrant and Australian women.

This study highlights the critical importance of healthcare provider recommendations to pregnant women, this is consistent with the current literature ${ }^{(15-17,23-26)}$. Of the reviewed determinants, recommendation to receive the vaccine demonstrated the greatest odds ratio to increase the vaccine uptake and was also most commonly self-identified as supporting antenatal vaccination.

System barriers were also a common theme that contributed to women not receiving vaccination. Nearly a quarter of all women who did not receive a pertussis vaccine reasoned that they ran out of time. Previous research has shown that health providers who recommend vaccination, yet are unable to provide the vaccine represent a system barrier ${ }^{(27)}$. The introduction of the point of care immunisation clinic in our study setting helped to eliminate this system barrier and likely contributed to high vaccine uptake rates.

This study has some limitations. First, our data relied upon women's accurate self-reports. Selfreport has been found to be a reasonable proxy for vaccine uptake in pregnant Australian women ${ }^{(28)}$ thus it is doubtful that this would greatly alter the findings of the study.

Secondly, women who are cared for by the Midwifery Group Practice may be discharged directly from the Birthing Unit. These women and any woman who birthed at home were not approached by researchers. This may have introduced some selection bias to the data. Past research has been inconsistent with a recent systematic review finding that women cared for by group midwifery services were not any more or less likely to receive antenatal vaccinations ${ }^{(21)}$, conversely a recent Australian study found that women receiving care via private midwifery practice were less likely to undergo antenatal vaccination ${ }^{(29)}$. It is therefore possible that the exclusion of some women from this group may impact on the data obtained. Thirdly, questionnaires were completed in a deidentified manner, which meant it was not possible to include a comparison of demographic characteristics for women who completed the survey and those who did not. A description of sociodemographic characteristics of the entire population of potentially eligible women has been included as an alternative. While these demographic data demonstrate that the groups are similar, this does not eliminate the possibility of selection bias associated with the use of a convenience sample. Our study determined that, with an uptake of $48.3 \%$, it is unlikely that women and their infants are sufficiently protected against seasonal influenza illness. The uptake of $82.9 \%$ for pertussis vaccine was greater than previous studies have found and suggests that uptake of the vaccine continues to trend upward for metropolitan centres in Australia, particularly those able to provide point of care access to vaccination.

Our results further strengthen the understanding that the recommendation of a healthcare provider is the most significant factor in determining women's acceptance of the vaccine. All clinicians caring for pregnant women should endeavour to include a discussion regarding the benefits of vaccination with their patients. System barriers to vaccination may be addressed by providing access to the vaccines in the antenatal clinic setting. 


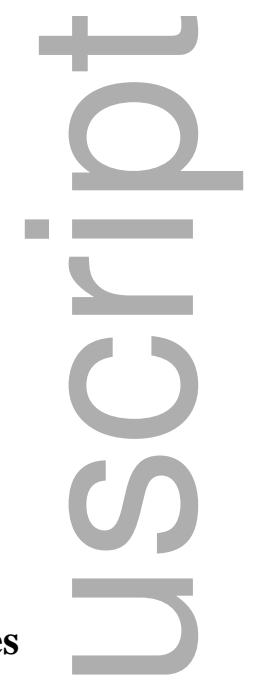

List of Figures

1. Figure 1. Rates of vaccination breakdown by month of birth

2. Figure 2. Thematic breakdown of vaccination decisions

\section{List of Tables}

1. Table 1. Socio-demographic characteristics

2. Table 2. Summary statistics and logistic regression of participants by vaccine status

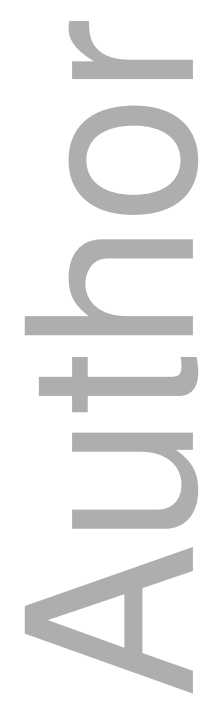

This article is protected by copyright. All rights reserved 


\section{References}

1. (ATAGI) ATAGol. The Australian Immunisation Handbook 10th ed (2016 update). Canberra: Australian Government Department of Health. 2016.

2. Beard FH. Pertussis immunisation in pregnancy: a summary of funded Australian state and territory programs. Commun Dis Intell Q Rep. 2015;39(3):E329-36.

3. McIntyre P, Wood N. Pertussis in early infancy: disease burden and preventive strategies. Curr Opin Infect Dis. $2009 ; 22(3): 215-23$.

4. Pertussis vaccines: WHO position paper - September 2015. Wkly Epidemiol Rec. 2015;90(35):433-58.

5. group WSpw. Background paper. SAGE April 20142014 Apr [cited 2017 February 17].

6. (ACIP) AColP. Updated Recommendations for Use of Tetanus Toxoid, Reduced Diphtheria Toxoid and Acellular Pertussis Vaccine (Tdap) in Pregnant Women and Persons Who Have or Anticipate Having Close Contact with an Infant Aged <12 Months. Morbidity and Mortality Weekly Report. 2011;Vol. 60:1424 - 6.

7. Amirthalingam $\mathrm{G}$, Andrews $\mathrm{N}$, Campbell H, Ribeiro S, Kara E, Donegan K, et al. Effectiveness of maternal pertussis vaccination in England: an observational study. Lancet. 2014;384(9953):1521-8.

8. Vaccines against influenza WHO position paper - November 2012. Wkly Epidemiol Rec. 2012;87(47):461-76.

9. Li-Kim-Moy J, Yin JK, Patel C, Beard FH, Chiu C, Macartney KK, et al. Australian vaccine preventable disease epidemiological review series: Influenza 2006 to 2015. Commun Dis Intell Q Rep. 2016;40(4):E482-e95.

10. Zaman K, Roy E, Arifeen SE, Rahman M, Raqib R, Wilson E, et al. Effectiveness of maternal influenza immunization in mothers and infants. N Engl J Med. 2008;359(15):1555-64.

11. coverage CfDCaPMv. Centers for Disease Control and Prevention 2017 [Available from: https://www.cdc.gov/vaccines/pregnancy/hcp-toolkit/maternal-vaccination-coverage.html.

12. Schuster M, Eskola J, Duclos P. Review of vaccine hesitancy: Rationale, remit and methods. Vaccine. 2015;33(34):4157-60.

13. Rowe SL, Perrett KP, Morey R, Stephens N, Cowie BC, Nolan TM, et al. Influenza and pertussis vaccination of women during pregnancy in Victoria, 2015-2017. Med J Aust. 2019;210(10):454-62.

14. Krishnaswamy S, Cheng AC, Wallace EM, Buttery J, Giles ML. Understanding the barriers to uptake of antenatal vaccination by women from culturally and linguistically diverse backgrounds: A cross-sectional study. Hum Vaccin Immunother. 2018;14(7):1591-8.

15. Bodeker B, Walter D, Reiter S, Wichmann O. Cross-sectional study on factors associated with influenza vaccine uptake and pertussis vaccination status among pregnant women in Germany. Vaccine. 2014;32(33):4131-9.

16. Hayles EH, Cooper SC, Sinn J, Wood N, Leask J, Skinner SR. Pertussis vaccination coverage among Australian women prior to childbirth in the cocooning era: a two-hospital, cross-sectional survey, 2010 to 2013. Aust N Z J Obstet Gynaecol. 2016;56(2):185-91.

17. Wilson RJ, Paterson P, Jarrett C, Larson HJ. Understanding factors influencing vaccination acceptance during pregnancy globally: A literature review. Vaccine. 2015;33(47):6420-9.

This article is protected by copyright. All rights reserved 
18. Mohammed H, Clarke M, Koehler A, Watson M, Marshall H. Factors associated with uptake of influenza and pertussis vaccines among pregnant women in South Australia. PLOS ONE. 2018;13(6):e0197867.

19. Division UNS. United Nations Geoshcemes https://unstats.un.org/unsd/methodology/m49/.

20. Braun V, Clarke V. Using thematic analysis in psychology. Qualitative Research in Psychology. 2006;3(2):77101.

21. Yuen CY, Tarrant M. Determinants of uptake of influenza vaccination among pregnant women - a systematic review. Vaccine. 2014;32(36):4602-13.

22. Krishnaswamy S, Wallace EM, Cheng AC, Buttery J, Giles ML. Protecting newborns from pertussis: The role of partner vaccination in the era of maternal immunization. Eur J Obstet Gynecol Reprod Biol. 2017;216:159-63.

23. Mak DB, Regan AK, Joyce S, Gibbs R, Effler PV. Antenatal care provider's advice is the key determinant of influenza vaccination uptake in pregnant women. Aust N Z J Obstet Gynaecol. 2015;55(2):131-7.

24. Myers KL. Predictors of maternal vaccination in the United States: An integrative review of the literature. Vaccine. 2016;34(34):3942-9.

25. Wiley KE, Cooper SC, Wood N, Leask J. Understanding pregnant women's attitudes and behavior toward influenza and pertussis vaccination. Qual Health Res. 2015;25(3):360-70.

26. Henninger ML, Irving SA, Thompson M, Avalos LA, Ball SW, Shifflett P, et al. Factors associated with seasonal influenza vaccination in pregnant women. J Womens Health (Larchmt). 2015;24(5):394-402.

27. Ding $\mathrm{H}$, Black $\mathrm{CL}$, Ball S, Donahue S, Fink RV, Williams WW, et al. Influenza Vaccination Coverage Among Pregnant Women--United States, 2014-15 Influenza Season. MMWR Morb Mortal Wkly Rep. 2015;64(36):1000-5.

28. Mak DB, Regan AK, Vo DT, Effler PV. Antenatal influenza and pertussis vaccination in Western Australia: a cross-sectional survey of vaccine uptake and influencing factors. BMC pregnancy and childbirth. 2018;18(1):416.

29. Regan AK, Mak DB, Hauck YL, Gibbs R, Tracey L, Effler PV. Trends in seasonal influenza vaccine uptake during pregnancy in Western Australia: Implications for midwives. Women and birth : journal of the Australian College of Midwives. 2016;29(5):423-9.

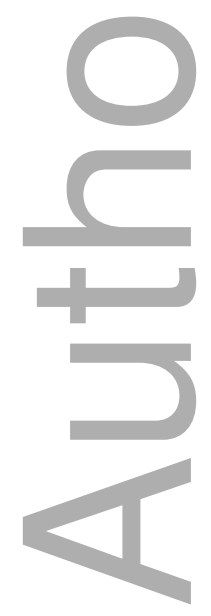




\section{Table 1. Socio-demographic characteristics}

\begin{tabular}{|c|c|c|}
\hline Study Factor & $\begin{array}{l}\text { Study Population, \% } \\
(\mathrm{n}=1305)\end{array}$ & $\begin{array}{l}\text { Eligible Population, \% } \\
(\mathrm{n}=5533)\end{array}$ \\
\hline \multicolumn{3}{|l|}{ Age Group } \\
\hline Less than 20 & $18(1.4 \%)$ & $96(1.7 \%)$ \\
\hline $20-25$ & $182(14.0 \%)$ & $950(17.2 \%)$ \\
\hline $26-30$ & $442(33.9 \%)$ & $1891(34.2 \%)$ \\
\hline $31-35$ & $432(33.1 \%)$ & $1753(31.7 \%)$ \\
\hline $36-4$ & $194(14.9 \%)$ & $711(12.8 \%)$ \\
\hline Over 4 & $37(2.8 \%)$ & $132(2.4 \%)$ \\
\hline \multicolumn{3}{|l|}{ Region of Origin } \\
\hline Australia & $560(42.9 \%)$ & $2201(39.8 \%)$ \\
\hline Southern Asia & $240(18.4 \%)$ & $1003(18.1 \%)$ \\
\hline South-Eastern Asia & $224(17.6 \%)$ & $914(16.5 \%)$ \\
\hline Central, Eastern and Western Asia & $48(3.7 \%)$ & $208(3.8 \%)$ \\
\hline The Americas & $23(1.7 \%)$ & $59(1.1 \%)$ \\
\hline Europe & $55(4.2 \%)$ & $210(3.8 \%)$ \\
\hline Africa & $63(4.8 \%)$ & $482(8.7 \%)$ \\
\hline $\begin{array}{l}\text { New Zealand and Pacific Island } \\
\text { Nations }\end{array}$ & Nations & $359(6.5 \%)$ \\
\hline Unknown/Unspecified & N/A & $97(1.8 \%)$ \\
\hline \multicolumn{3}{|l|}{$\begin{array}{l}\text { ATSI Status - Identified as Aboriginal or } \\
\text { Torres Strait Islander }\end{array}$} \\
\hline Yes & $10(1.7 \%)$ & $43(0.8 \%)$ \\
\hline No & $1295(98.3 \%)$ & $5490(99.2 \%)$ \\
\hline \multicolumn{3}{|l|}{ Primary Care Provider Type in Pregnancy } \\
\hline Hospital Antenatal Clinic & $927(71.0 \%)$ & $\begin{array}{l}\text { Data not } \\
\text { available }\end{array}$ \\
\hline Midwife Clinic affiliated with & $263(20.2 \%)$ & \\
\hline \multicolumn{3}{|l|}{ Hospital } \\
\hline Antenatal Clinic at Another Hospital & $26(2.0 \%)$ & \\
\hline General Practitioner & $72(5.5 \%)$ & \\
\hline Private Obstetrician & $14(1.0 \%)$ & \\
\hline Private Midwife & $3(0.2 \%)$ & \\
\hline \multicolumn{3}{|l|}{ Gestation at Delivery } \\
\hline $32^{+0}-33^{+6}$ weeks & $47(3.6 \%)$ & $55(1.0 \%)$ \\
\hline $34^{+0}-35^{+6}$ weeks & $60(4.6 \%)$ & $138(4.1 \%)$ \\
\hline
\end{tabular}

This article is protected by copyright. All rights reserved 


\begin{tabular}{|c|c|c|}
\hline $36^{+0}-37^{+6}$ weeks & $159(12.8 \%)$ & $810(14.6 \%)$ \\
\hline $38^{+0}-39^{+6}$ weeks & $694(53.2 \%)$ & $2963(53.6 \%)$ \\
\hline$>40$ weeks & $330(25.3 \%)$ & $1567(28.3 \%)$ \\
\hline \multicolumn{3}{|l|}{ Parity } \\
\hline \multirow{3}{*}{$\begin{array}{l}1 \\
2 \\
3+\end{array}$} & $577(44.2 \%)$ & $2222(40.2 \%)$ \\
\hline & $464(35.6 \%)$ & $1910(34.5 \%)$ \\
\hline & $264(20.2 \%)$ & $1401(25.3 \%)$ \\
\hline \multicolumn{3}{|c|}{ Language Questionnaire Was Completed In } \\
\hline \multirow{2}{*}{$\begin{array}{l}\text { English } \\
\text { Vietnamese }\end{array}$} & $1273(97.6 \%)$ & \multirow[t]{6}{*}{ N/A } \\
\hline & $26(2.0 \%)$ & \\
\hline Arabic & $1(0.1 \%)$ & \\
\hline Burme & $2(0.2 \%)$ & \\
\hline Simplified Chinese & $2(0.2 \%)$ & \\
\hline Punjabi & $1(0.1 \%)$ & \\
\hline
\end{tabular}

$\star$ rounded percentages may not total $100 \%$

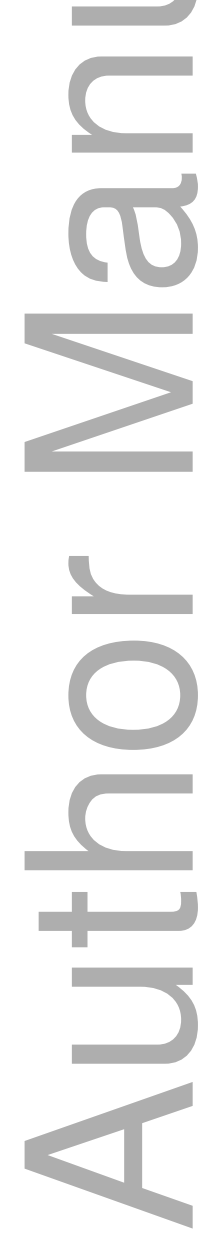


Table 2. Summary statistics and logistic regression of participants by vaccine status

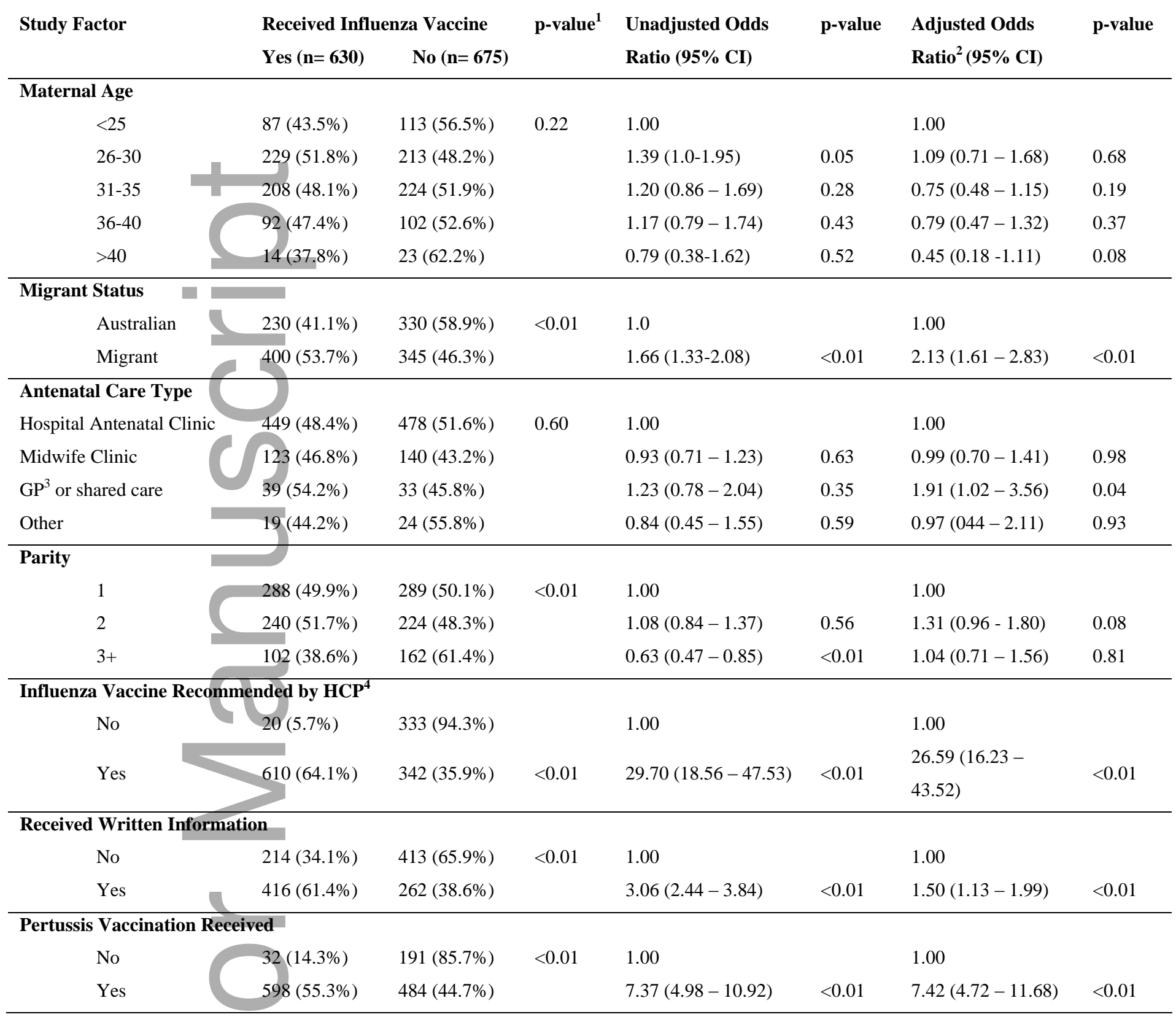




\begin{tabular}{|c|c|c|c|c|c|c|c|}
\hline \multirow[t]{2}{*}{ Study Factor } & \multicolumn{2}{|c|}{ Received Pertussis Vaccine } & \multirow{2}{*}{ p-value ${ }^{1}$} & \multirow{2}{*}{$\begin{array}{l}\text { Unadjusted Odds } \\
\text { Ratio }(95 \% \text { CI })\end{array}$} & \multirow[t]{2}{*}{ p-value } & \multirow{2}{*}{$\begin{array}{l}\text { Adjusted Odds } \\
\text { Ratio }^{2}(95 \% \text { CI })\end{array}$} & \multirow[t]{2}{*}{ p-value } \\
\hline & Yes (n- 1082) & No (n- 223) & & & & & \\
\hline \multicolumn{8}{|l|}{ Maternal Age } \\
\hline $26-30$ & $372(84.2 \%)$ & $70(15.8 \%)$ & & $1.69(1.11-2.53)$ & 0.01 & $1.79(1.03-3.09)$ & 0.04 \\
\hline $31-35$ & $379(87.7 \%)$ & $53(12.3 \%)$ & & $2.26(1.46-3.48)$ & $<0.01$ & $4.10(2.25-7.48)$ & $<0.01$ \\
\hline$>40$ & $28(75.7 \%)$ & $9(24.3 \%)$ & & $0.98(0.43-2.33)$ & 0.97 & $1.68(0.59-4.78)$ & 0.33 \\
\hline \multicolumn{8}{|l|}{ Migrant Status } \\
\hline Australian & $483(86.3 \%)$ & $77(13.7 \%)$ & $<0.01$ & 1.0 & & 1.0 & \\
\hline Migrant & $599(80.4 \%)$ & $146(19.6 \%)$ & & $0.65(0.48-0.88)$ & $<0.01$ & $0.50(0.33-0.75)$ & $<0.01$ \\
\hline $\mathrm{GP}^{3}$ or shared care & $52(86.1 \%)$ & $10(13.9 \%)$ & & $1.30(0.65-2.59)$ & 0.45 & $0.77(0.33-1.80)$ & 0.55 \\
\hline Other & $36(83.7 \%)$ & $7(16.3 \%)$ & & $1.08(0.47-2.47$ & 0.85 & $1.20(0.38-3.8)$ & 0.76 \\
\hline \multicolumn{8}{|l|}{ Parity } \\
\hline 1 & $24(90.8 \%)$ & $53(9.2 \%)$ & $<0.01$ & 1.0 & & 1.0 & \\
\hline 2 & $386(83.2 \%)$ & $78(16.8 \%)$ & & $0.50(0.34-0.73)$ & $<0.01$ & $0.38(0.24-0.61)$ & $<0.01$ \\
\hline $3+$ & $172(65.2 \%)$ & $92(34.8 \%)$ & & $0.19(1.2-0.28)$ & $<0.01$ & $0.18(0.11-0.31)$ & $<0.01$ \\
\hline \multicolumn{8}{|c|}{ Pertussis Vaccine Recommended by $\mathrm{HCP}^{4}$} \\
\hline No & $12(11.4 \%)$ & $93(88.6 \%)$ & $<0.01$ & 1.0 & & 1.0 & \\
\hline Yes & $1070(89.2 \%)$ & $130(10.8 \%)$ & & $\begin{array}{l}63.79(34.04- \\
119.55)\end{array}$ & $<0.01$ & $\begin{array}{l}41.78(20.03- \\
87.17)\end{array}$ & $<0.01$ \\
\hline \multicolumn{8}{|l|}{ Received Influenza } \\
\hline \multicolumn{8}{|l|}{ Vaccine } \\
\hline No & $484(71.7 \%)$ & $191(28.3 \%)$ & $<0.01$ & 1.0 & & 1.0 & \\
\hline Yes & $598(94.9 \%)$ & $32(5.1 \%)$ & & 7.37 (4.98- 10.92) & $<0.01$ & $9.18(5.59-15.08)$ & $<0.01$ \\
\hline
\end{tabular}

Table 2. 1. Statistical significance calculated with Fisher's Exact Test.

2. Odds ratios adjusted for all other variables displayed in table.

3. General practitioner.

4. Healthcare provider.

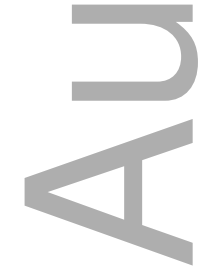

This article is protected by copyright. All rights reserved 


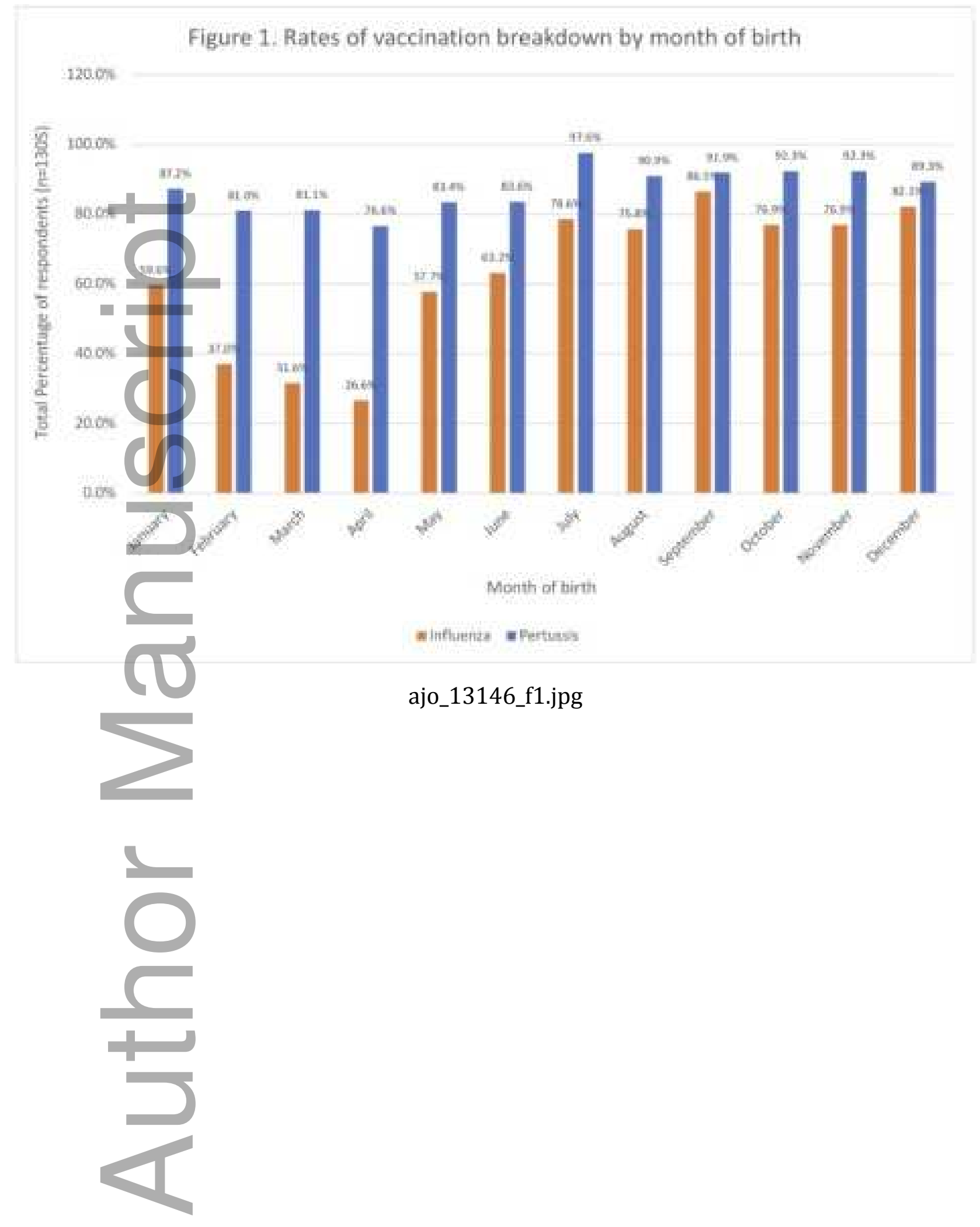




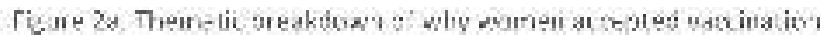
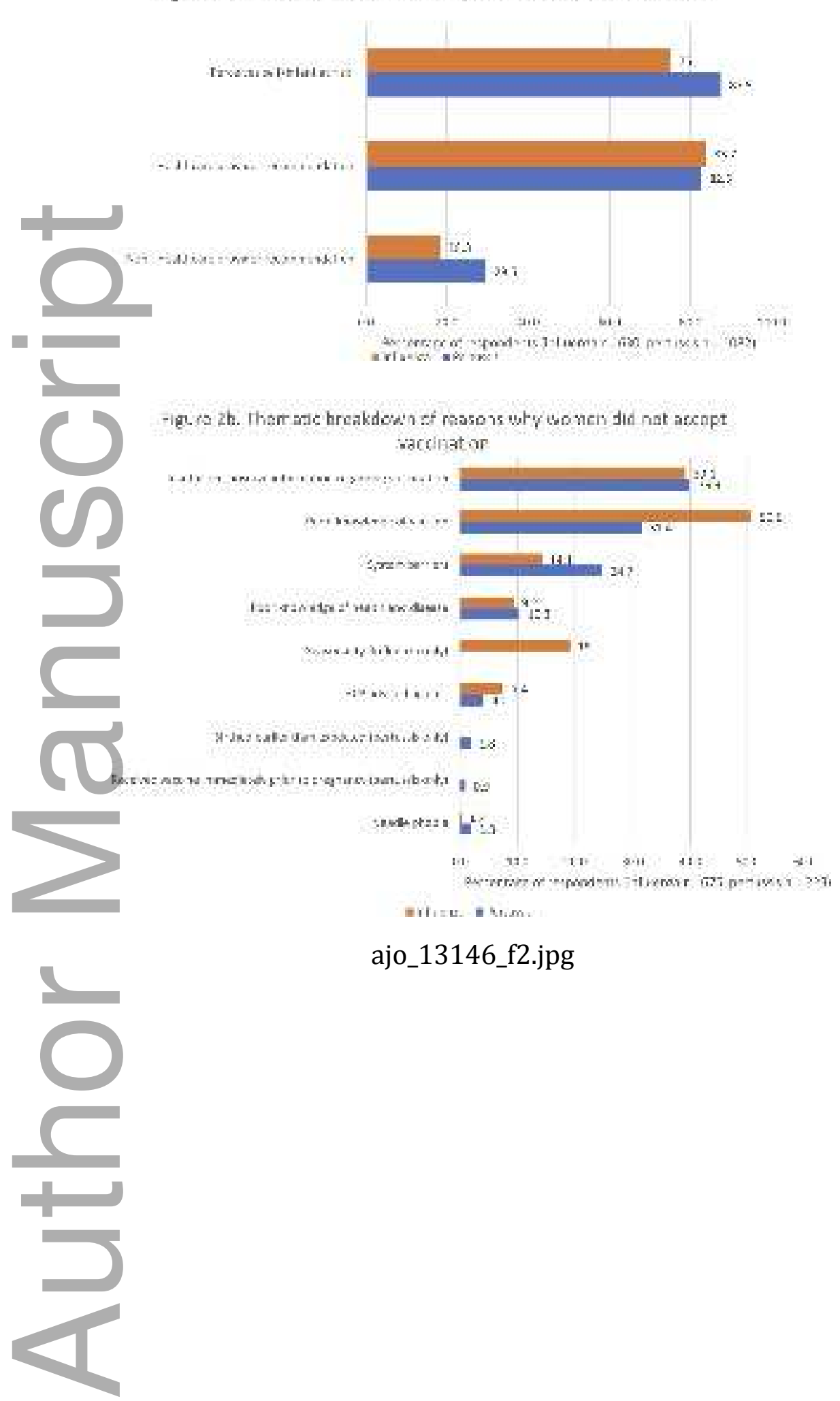

ajo_13146_f2.jpg 


\section{University Library}

\section{- M M N E R VA A gateway to Melbourne's research publications}

Minerva Access is the Institutional Repository of The University of Melbourne

Author/s:

Moir, D;Gunter, K;Lynch, L-A;Vogrin, S;Said, J

Title:

Antenatal vaccine uptake: A cross-sectional study investigating factors influencing women's choices in pregnancy

Date:

2020-03-23

\section{Citation:}

Moir, D., Gunter, K., Lynch, L. -A., Vogrin, S. \& Said, J. (2020). Antenatal vaccine uptake: A cross-sectional study investigating factors influencing women's choices in pregnancy. AUSTRALIAN \& NEW ZEALAND JOURNAL OF OBSTETRICS \& GYNAECOLOGY, 60 (5), pp.729-737. https://doi.org/10.1111/ajo.13146.

Persistent Link:

http://hdl.handle.net/11343/275562 